\title{
ANTI-INFLAMMATORY ACTIVITY OF Blutaparon portulacoides ETHANOLIC EXTRACT AGAINST THE INFLAMMATORY REACTION INDUCED BY Bothrops jararacussu VENOM AND ISOLATED MYOTOXINS BthTX-I AND II
}

\section{Pereira IC (1), Barbosa AM (1), Salvador MJ (2), Soares AM (3), Ribeiro W (1), Cogo JC (1), Zamuner SR (4)}

(1) Laboratory of Inflammation, Institute of Research and Development, Vale do Paraíba University, UNIVAP, São José dos Campos, São Paulo State, Brazil; (2) Institute of Biology, Department of Vegetal Biology, Pharmacy Course, State University of Campinas, UNICAMP, Campinas, São Paulo State, Brazil; (3) Department of Clinical, Toxicological and Bromatological Analysis, School of Pharmaceutical Sciences of Ribeirão Preto, University of São Paulo, USP, Ribeirão Preto, São Paulo State, Brazil. (4) School of Applied Sciences, State University of Campinas, UNICAMP, Limeira, São Paulo State, Brazil.

ABSTRACT: This article reports the anti-inflammatory effect of Blutaparon portulacoides (B. portulacoides), specifically the ethanolic extract of its aerial parts, on the edema formation and leukocyte influx caused by Bothrops jararacussu ( $B$. jararacussu) snake venom and Bothropstoxin-I and II (BthTX-I and II) isolated from this venom as an alternative treatment for Bothrops snakebites. The antiinflammatory effect of $B$. portulacoides ethanolic extract was compared with an animal group pretreated with dexamethasone. B. portulacoides ethanolic extract significantly inhibited paw edema induced by $B$. jararacussu venom and by BthTX-I and II. Also, results demonstrated that the extract caused a reduction of the leukocyte influx induced by BthTX-I. However, the extract was not capable of inhibiting the leukocyte influx induced by the venom and by BthTX-II. In conclusion, these results suggest that the ethanolic extract of this plant possess components able to inhibit or inactivate toxins present in $B$. jararacussu venom, including its myotoxins, responsible for the edema formation. However, the leukocyte migration caused by the venom and BthTX-II was not inhibited by the plant, probably due to the different mechanisms involved in the edema formation and leukocyte influx. This is the first report of $B$. portulacoides extract as anti-inflammatory against snake venoms and isolated toxins.

KEY WORDS: Bothrops jararacussu, myotoxins, inflammation, edema, Blutaparon portulacoides.

CONFLICTS OF INTEREST: There is no conflict.

CORRESPONDENCE TO:

STELLA REGINA ZAMUNER, rua Pedro Zacarias, 1300, Limeira, SP, Brasil. Phone: +55 1937016675 . Email: szamuner@unicamp.br. 
Pereira IC et al. Anti-inflammatory activity of Blutaparon portulacoides ethanolic extract against the inflammatory reaction induced by Bothrops jararacussu venom and isolated myotoxins BthTX-I and II. J Venom Anim Toxins incl Trop Dis. 2009;15(3):528

\section{INTRODUCTION}

Plants have often been used by humans, sometimes successfully, against numerous diseases caused by different pathological agents. Pharmacological studies have demonstrated that the extracts and fractions from some of these plants used in traditional medicine possess anti-inflammatory, antiviral and antiophidian properties that constitute an alternative for ophidic accident treatment, displaying a large diversity of chemical compounds with several pharmacological activities of medicalscientific interest (1-7). The antiophidian activity of several plant species in general use in some Brazilian communities has been investigated (2, 8-15).

B. portulacoides, belongs to the Amaranthaceae family, Magnoliopsida class, Caryophyllales order, characterized by A. L. Jussieu in 1789. Herbaceous plants of annual and permanent growth are established predominantly in temperate, subtropical and tropical regions. In Brazil they occur mainly in the Atlantic bush (16). In folk medicine, B. portulacoides has been employed for the treatment of leucorrhea (17). In an experimental model, the crude extract presented trypanocidal and leishmanicidal activity in vitro, and also antimicrobial activity (18).

Snake venoms are complex mixtures of proteins including phospholipases $A_{2}$, myotoxins, hemorrhagic metalloproteases and additional proteolytic enzymes, cytotoxins, cardiotoxins, among others. The pathophysiology of snake envenomation involves a complex series of events that depend on the combined action of these venom components (19). The venom of the snake $B$. jararacussu causes symptoms similar those provoked by other Bothrops species including hemorrhage $(4,20,21)$, myonecrosis $(22,23)$ and edema $(24)$. The local effects caused by $B$. jararacussu venom are due to, at least in part, its myotoxin content (25). Two myotoxins with PLA $_{2}$ structure, BthTX-I and BthTX-II, have been isolated and characterized from $B$. jararacussu snake venom $(26,27)$. These proteins can be classified into two categories: the Asp49 PLA 2 , catalytically active, and Lys49 PLA 2 , devoid of significant catalytic activity upon artificial substrate (28-30). BthTX-I (Lys 49) induces several pharmacological effects which include edema, mastocyte degranulation, irreversible blockade of muscle contraction, liposome disruption, and cytotoxicity upon muscle and endothelial cells $(26,27,31)$. BthTX-II (Asp 49) induces edema and leukocyte migration $(32,33)$. 
In this study, we examined the anti-inflammatory activity of $B$. portulacoides ethanolic extract against edema formation and leukocyte influx induced by $B$. jararacussu snake venom and its main isolated myotoxins.

\section{MATERIALS AND METHODS}

\section{Plant Material}

Aerial parts of $B$. portulacoides (Amaranthaceae) were collected at Restinga de Maricá, Rio de Janeiro, RJ, Brazil, in December 2002 and identified by Prof. Dr. Josafá Carlos de Siqueira (PUC-Rio, Rio de Janeiro, RJ). A voucher specimen was deposited at the Herbarium of the Department of Biology, Ribeirão Preto School of Philosophy, Sciences and Literature, University of São Paulo, SP, Brazil (registration number SPSFR 02961).

\section{Preparation of Plant Extract}

The powdered, air-dried aerial parts of the plant $(1000 \mathrm{~g})$ were extracted exhaustively by maceration at room temperature with hexane and ethanol successively, in the powder/solvent mass ratio of 1:20 (mass/volume). The biomass was filtered from the extracts and the solvents were removed under vacuum in a rotary evaporator (below $40^{\circ} \mathrm{C}$ ), to obtain the hexanic (yield $=6 \mathrm{~g}$ ) and ethanolic (yield $=90 \mathrm{~g}$ ) crude extracts.

\section{Venom and Myotoxins}

Lyophilized crude venom of Bothrops jararacussu were supplied by Dr. José Carlos Cogo, Serpentariun of CEN (Nature Center of Study), UNIVAP, São José dos Campos, SP, Brazil. Myotoxins bothropstoxins-I (BthTX-I) and bothropstoxin-II (BthTX-II) were supplied by Dr. Andreimar M. Soares, from the University of São Paulo, USP, Ribeirão Preto, SP, Brazil. BthTX-I and II were isolated and purified as previously described $(26,27,34)$.

\section{Animals}

All animal care was in accordance with the guidelines of the Brazilian College for Animal Experimentation (COBEA) and approved by the Committee for Ethics in Animal Research of UNIVAP, under number A117/2007/CEP. Male, Swiss mice (22$25 \mathrm{~g}$ ) (45 days old) were used through the experiment, randomly divided into six 
groups of five animals each. Animals were kept in plastic cages, with water and food ad libitum, and maintained under controlled temperatures $\left(26^{\circ} \mathrm{C}\right)$ and on a 12 hour light/dark cycle.

\section{Dose-response Curve}

To determine the optimum dose of $B$. portulacoides the animals received intraperitoneal (i.p.) injections of $B$. portulacoides ethanolic extract at the doses 100 , 250 or $500 \mathrm{mg} / \mathrm{kg}$ (constant volume of $100 \mu \mathrm{L}$ ), one hour before venom $(0.1 \mathrm{mg} / \mathrm{kg})$ and the edema was measured.

\section{Evaluation of Paw Edema}

Fifty microliters of sterile saline containing $0.1 \mathrm{mg} / \mathrm{kg}$ of Bothrops jararacussu venom or $0.4 \mathrm{mg} / \mathrm{kg}$ of myotoxins (BthTX-I and BthTX-II) was injected in the subplantar region of the right hind paw in a total volume of $50 \mu \mathrm{L}$. The left hind paw received an equal volume of sterile saline alone and served as control. The volumes of both hind paws were measured by plethysmometry (Plethysmometer model 7140®, Ugo Basile, Italy) before and 15, 30 minutes, 1, 2, 4 and 6 hours after venom or myotoxins administration according to the method describe by Van Arman et al. (35). The edema was expressed as the percentage increase in the volume of the treated (right) paw relative to that of control (left) paw at each time interval. As anti-inflammatory control we used dexamethasone $(1 \mathrm{mg} / \mathrm{kg})$.

\section{Evaluation of Leukocytes Influx in the Peritoneal Cavity}

Bothrops jararacussu venom $(0.2 \mathrm{mg} / \mathrm{kg})$ or myotoxins $(0.8 \mathrm{mg} / \mathrm{kg}$ BthTX-I or 0.4 $\mathrm{mg} / \mathrm{kg}$ BthTX-II) dissolved in $1 \mathrm{ml}$ of sterile saline were injected i.p.. Groups of animals were killed six hours after the injections and the inflammatory exudates were withdrawn after washing the peritoneal cavity. Leukocytes were harvested by washing cavities with $2 \mathrm{~mL}$ of saline containing heparin $(5 \mathrm{U} / \mathrm{mL})$. Aliquots of the washes were used to determine total cell counts in a Neubauer chamber after dilution in Turk solution ( $0.2 \%$ crystal violet dye in $30 \%$ acetic acid). Differential leukocyte counts were performed on stained Instant Prov. 


\section{Statistical Analysis}

Mean and standard deviation were calculated for each group. To establish whether the difference between the mean values of any two experimental groups was significant the Student's t-test was performed, using a statistical significance level of $p<0.05$. When more than two groups were compared a two-way analysis of variance was applied, followed by the Tukey-Kramer test.

\section{RESULTS}

Effects of $B$. portulacoides Ethanolic Extract on Edema Formation Induced by Bothrops jararacussu Venom

Intraplantar injection of $B$. jararacussu venom $(0.1 \mathrm{mg} / \mathrm{kg})$ caused time-dependent paw-volume increases. Edema formation peaked one hour after injection of $B$. jararacussu venom (Figure 1).

To determine the optimum extract dose for inhibiting edema induced by $B$. jararacussu venom, animals were pretreated with 100, 250 or $500 \mathrm{mg} / \mathrm{kg}$, i.p., of $B$. portulacoides one hour before the injection of $0.1 \mathrm{mg} / \mathrm{kg}$ of venom (Figure 1). The dose of $100 \mathrm{mg} / \mathrm{kg}$ showed a small, non-significant reduction of edema. The extract doses of 250 and $500 \mathrm{mg} / \mathrm{kg}$ showed a significant reduction of the edematogenic effect $\left({ }^{*} p<0.05\right)$ in comparison to the edema induced by the venom. B. portulacoides at $500 \mathrm{mg} / \mathrm{kg}$ inhibited the edema formation by $53 \%, 36 \%, 35 \%, 37 \%$ and $39 \%, 30$ minutes, 1, 2, 4 and 6 hours, respectively, whereas the dose of $250 \mathrm{mg} / \mathrm{kg}$, in the same periods of time, presented reductions of $13 \%, 20 \%, 19 \%, 20.6 \%, 28.5 \%$. These results were comparable with dexamethasone. From these findings, a dose of $500 \mathrm{mg} / \mathrm{kg}$ i.p. of B. portulacoides was used in the subsequent studies. 


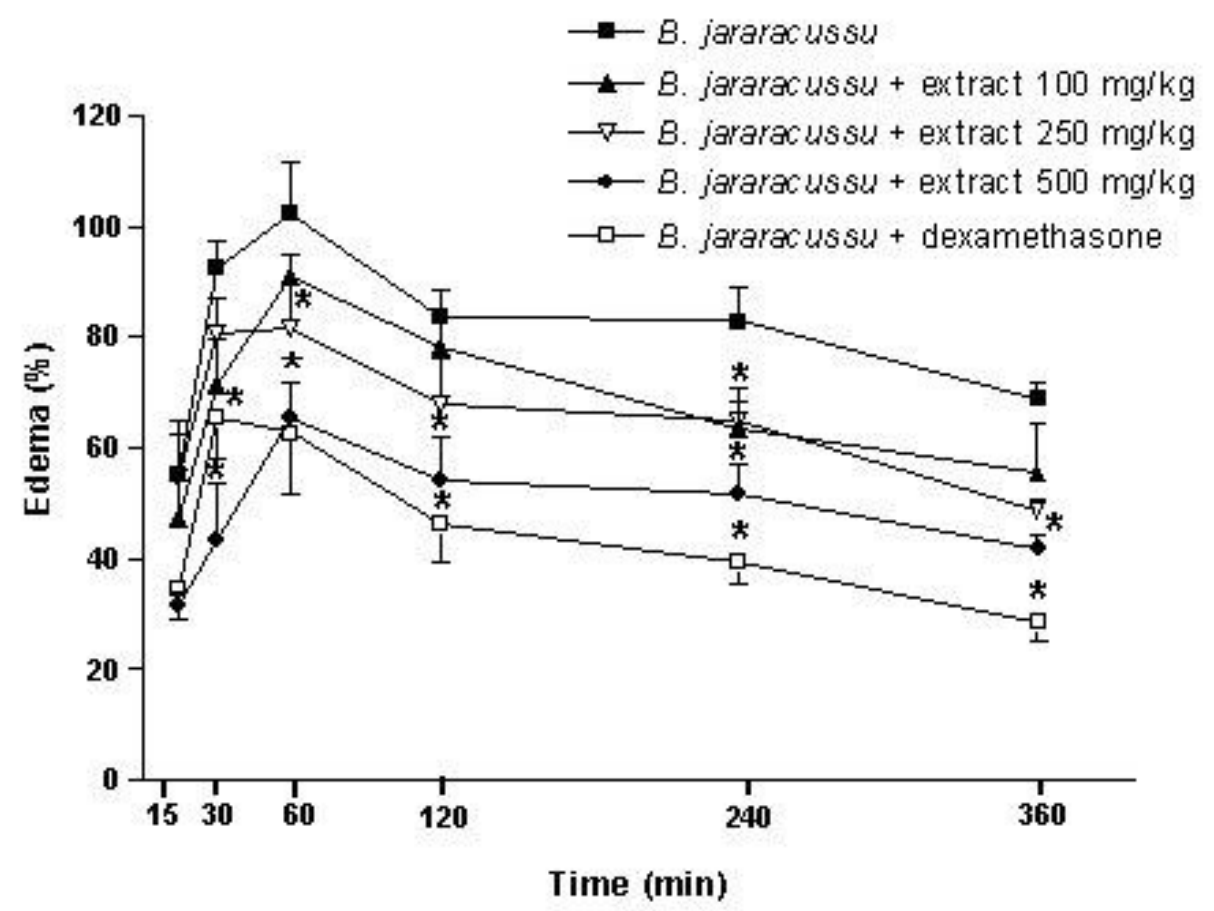

Figure 1. Antiedematogenic effect of the ethanolic extract from the B. portulacoides aerial parts on paw edema induced by $B$. jararacussu venom. Mice injected with $B$. jararacussu venom $(0.1 \mathrm{mg} / \mathrm{kg})$, were pretreated with the extract $(100,250,500$ $\mathrm{mg} / \mathrm{kg}$, i.p.) one hour before the venom. The other animal group was pretreated with dexamethasone (1 $\mathrm{mg} / \mathrm{kg}$, i.p.). Edema was evaluated plethysmographically at various time intervals (15, 30 minutes, 1, 24 and 6 hours) after venom injection and was expressed as the percentage of increase in the volume of the right as compared to the left footpad. Results are presented as mean \pm SEM $(n=5) .{ }^{*} p<0.05$.

\section{Effects of B. portulacoides Ethanolic Extract on Edema Formation Induced by Bothropstoxin I and II}

The edema formation was evaluated after the intraplantar injection of BthTX-I or BthTX-II isolated from the $B$. jararacussu venom at the dose of $0.4 \mathrm{mg} / \mathrm{kg}$ or saline (control). The kinetics of edema formation was evaluated 15, 30 minutes, 1, 2, 4 and 6 hours after the BthTX-I or BthTX-II injection. BthTX-I provoked edema formation that started 15 minutes after its injection and was elevated for 6 hours. The edema induced by BthTX-II had started by 15 minutes after its intraplantar application, followed by a peak at one hour and diminution in subsequent hours (Figure 2). The 
Pereira IC et al. Anti-inflammatory activity of Blutaparon portulacoides ethanolic extract against the inflammatory reaction induced by Bothrops jararacussu venom and isolated myotoxins BthTX-I and II. J Venom Anim Toxins incl Trop Dis. 2009;15(3):533

potential of the antiedematogenic effect of the extract, applied through the intraperitoneal (i.p) route, demonstrated that the BthTX-I extract caused a significant reduction of the edematogenic effect from 30 minutes of its administration, producing reductions of $48.2 \%, 38.9 \%, 36.9 \%, 45.1 \%$ and $45.0 \%$ at 30 minutes, $1,2,4$ and 6 hours, respectively (Figure 2A). For BthTX-II, the significant reduction of the edematogenic effect had started by 15 minutes, yielding respective reductions of $50.1 \%, 37.9 \%, 61.8 \%, 36.1 \%, 43.1 \%, 28.2 \%$ at 15 minutes, 30 minutes, $1,2,4$ and 6 hours (Figure 2B).
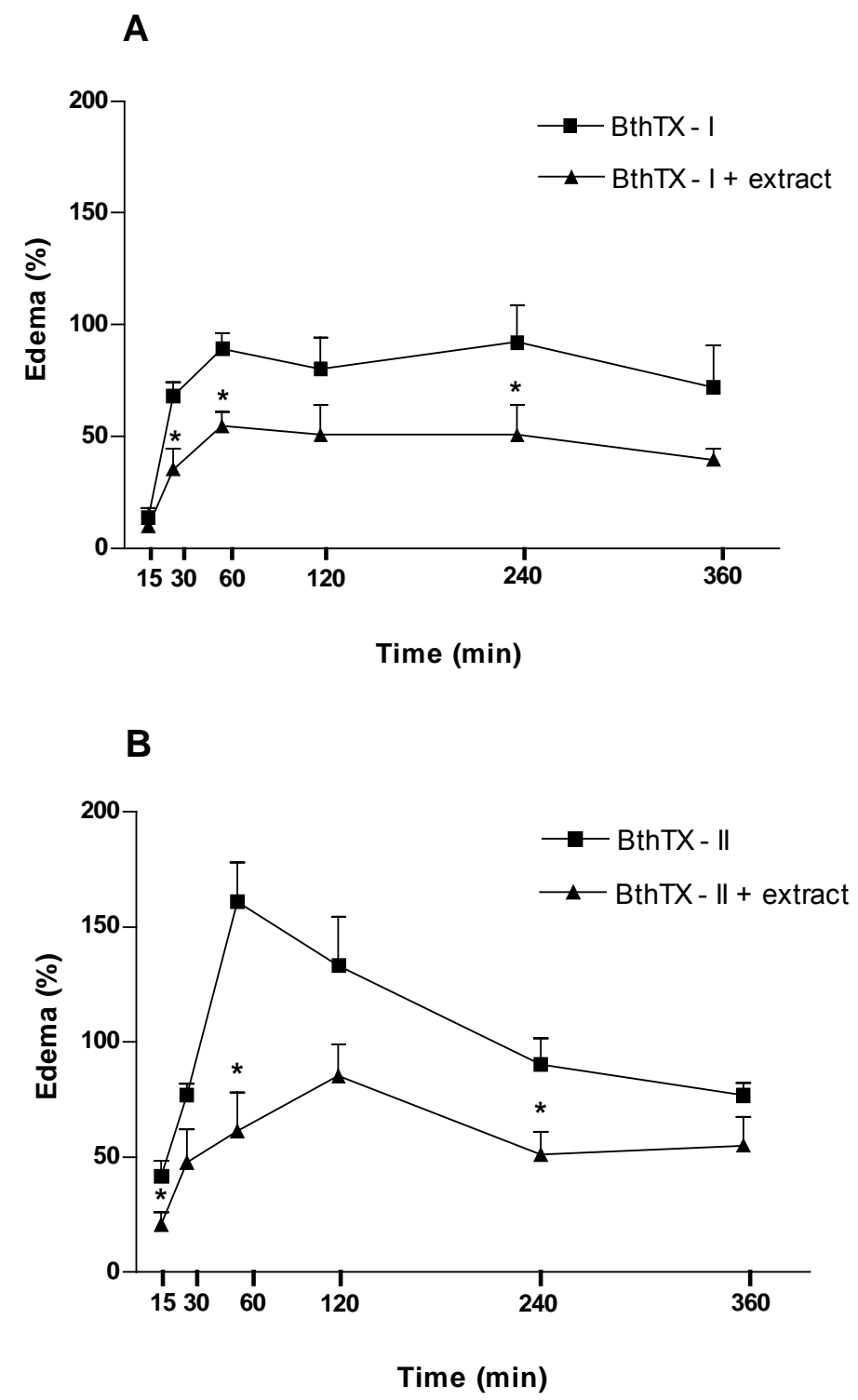

Figure 2. Effects of ethanolic extract from the $B$. portulacoides aerial parts on edema formation induced by BthTX-I and BthTX-II. (A) BthTX-I and (B) BthTX-II. Mice were pretreated with the $B$. portulacoides ethanolic extract $(500 \mathrm{mg} / \mathrm{kg}$, i.p.) one hour 
before BthTX-I or II injection. Then, after extract administration groups of mice were injected in the right paw with BthTX-I or BthTX-II $(0.4 \mathrm{mg} / \mathrm{kg})$ and saline (control) in the left paw. Edema was evaluated plethysmographically at various time intervals after myotoxin injection and expressed as the percentage increase in the volume of the right footpad as compared to the left footpad. Results are presented as mean \pm $\operatorname{SEM}(n=5) .{ }^{*} p<0.05$.

\section{Effects of B. portulacoides Ethanolic Extract on Leukocyte Migration Induced by Bothrops jararacussu Venom}

The leukocyte migration into the peritoneal cavity was evaluated six hours after i.p. injection of $0.2 \mathrm{mg} / \mathrm{kg}$ of the $B$. jararacussu venom. As shown in Figure 3 A, i.p. injection of $B$. portulacoides followed by i.p. injection of $B$. jararacussu caused accumulation of $8,430 \pm 1037 \times 10^{3} / \mathrm{mL}$ leukocytes, which did not differ from that of venom alone $\left(7,900 \pm 604 \times 10^{3} / \mathrm{mL}\right)$. Differential leukocyte counts are displayed in Figures $3 \mathrm{~B}$ and $3 \mathrm{C}$. An influx of polymorphonuclear (PMN) and mononuclear (MN) cells was induced by the venom (PMN: $6020 \pm 768 \times 10^{3} / \mathrm{mL}$; MN: $1879 \pm 340 \times$ $10^{3} / \mathrm{mL}$ ). PMN and MN influxes caused by $B$. jararacussu were not inhibited by the $B$. portulacoides extract (PMN: 6,541 $\pm 948 \times 10^{3} / \mathrm{mL}$; MN: $1,741 \pm 531 \times 10^{3} / \mathrm{mL}$ ) when compared with venom alone. 

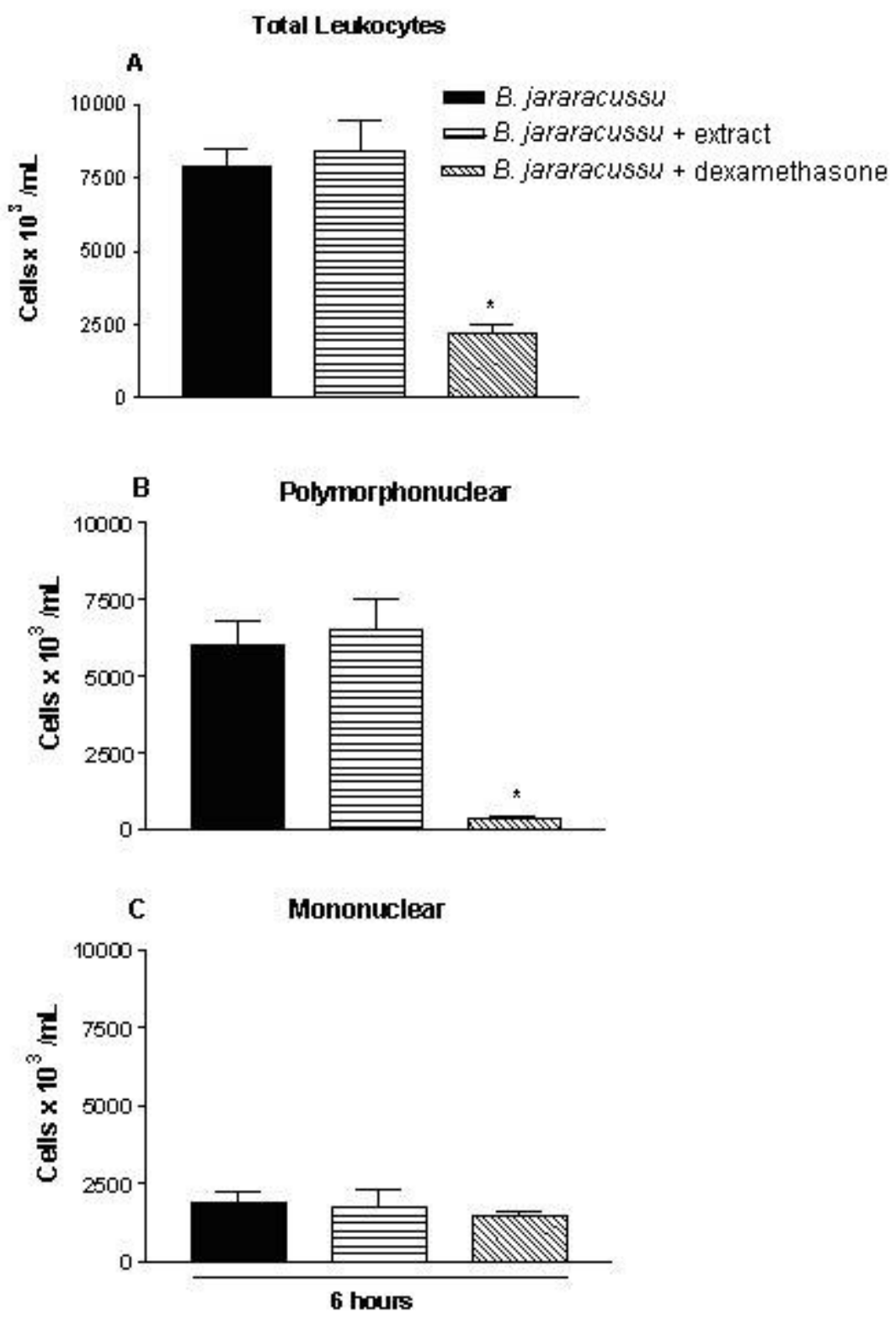

Figure 3. The effect of $B$. portulacoides ethanolic extract on leukocyte influx into the peritoneal cavity induced by $B$. jararacussu venom. Mice injected with $B$. jararacussu venom $(0.4 \mathrm{mg} / \mathrm{kg}$, i.p) dissolved in $1 \mathrm{~mL}$ of sterile saline and were pretreated with the extract (500 $\mathrm{mg} / \mathrm{kg}$, i.p.). Another group was pretreated with dexamethasone (1 $\mathrm{mg} / \mathrm{kg}$ i.p.). Total leukocytes (A), PMN cells (B) and MN cells (C). Mice were killed after six hours and inflammatory exudates were withdrawn after washing the peritoneal cavity. Results are presented as mean $\pm \operatorname{SEM}(n=5) .{ }^{*} p<0.05$. 


\section{Effects of $B$. portulacoides Ethanolic Extract on Leukocyte Migration Induced by BthTX-I and II}

Leukocyte migration into the peritoneal cavity was evaluated six hours after i.p. injection of BthTX-I or BthTX-II $(0.8 \mathrm{mg} / \mathrm{kg}$ of BthTX-I or $0.4 \mathrm{mg} / \mathrm{kg}$ of BthTX-II). Figure $4 \mathrm{~A}$ demonstrates the number of total leukocytes in the peritoneal cavity of the animals that had received BthTX-I or BthTX-II injection and those pretreated with 500 $\mathrm{mg} / \mathrm{kg}$ of the extract. BthTX-I and BthTX-II caused a significant and similar leukocyte influx into the peritoneal cavity. The extract treatment significantly inhibited the induced leukocyte influx caused by BthTX-I. However, the leukocyte influx induced by BthTX-II was not inhibited by the extract. The differential leukocyte counts in the peritoneal cavity six hours after the i.p BthTX-I or BthTX-II injection are shown in Figures $4 \mathrm{~B}$ and $4 \mathrm{C}$. Figure $4 \mathrm{~B}$ shows an important $\mathrm{PMN}$ cell influx, with predominance of neutrophils, after the injection of both myotoxins. The $B$. portulacoides extract inhibited PMN influx induced by BthTX-I, but not by BthTX-II (Figure 4B). Figure 4C shows the number of MN leukocytes, BthTX-II provoked significantly higher influx of these cells when compared with the BthTX-I. 
Pereira IC et al. Anti-inflammatory activity of Blutaparon portulacoides ethanolic extract against the inflammatory reaction induced by Bothrops jararacussu venom and isolated myotoxins BthTX-I and II. J Venom Anim Toxins incl Trop Dis. 2009;15(3):537
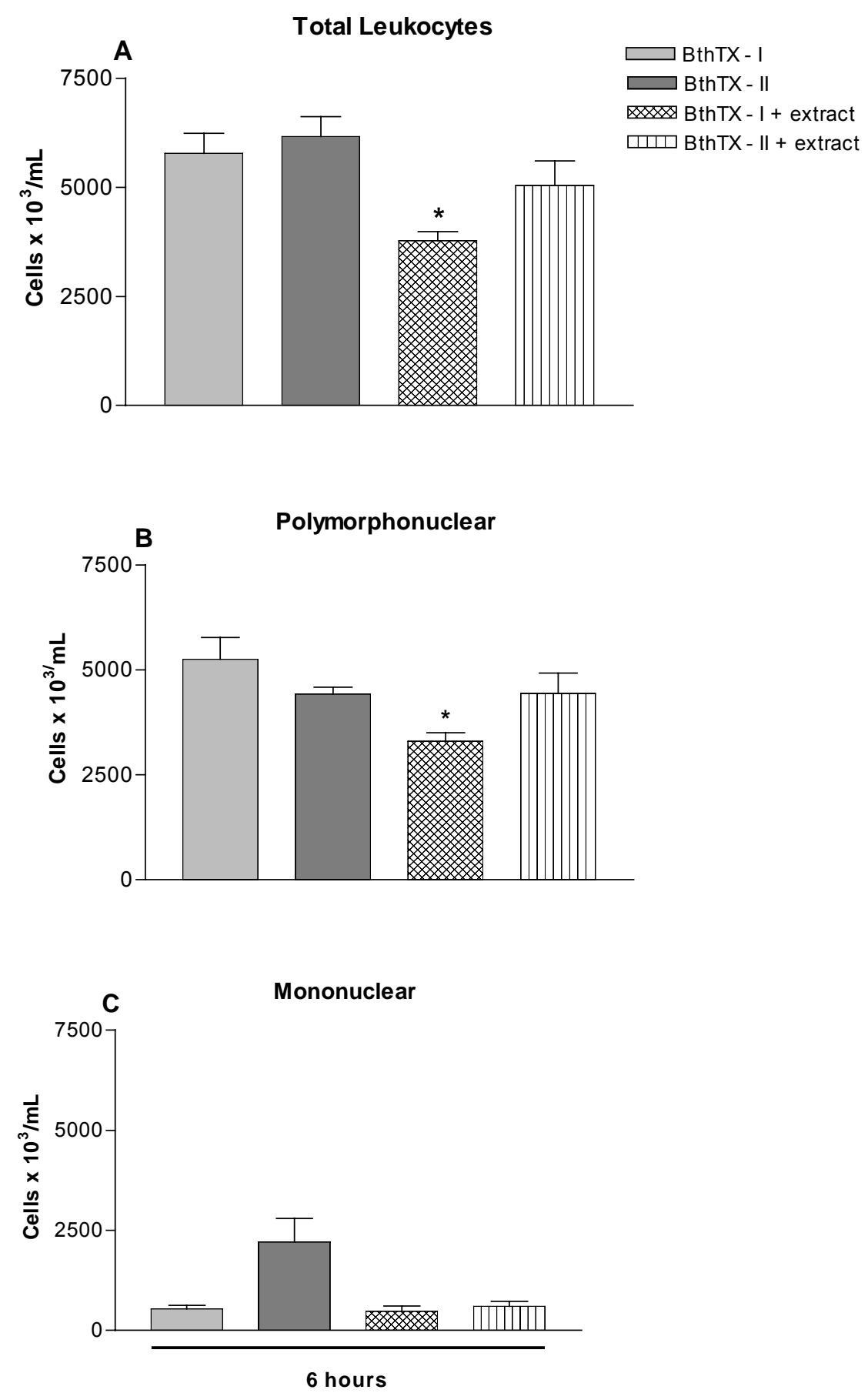

Figure 4. The effect of $B$. portulacoides ethanolic extract on leukocyte influx into the peritoneal cavity induced by two myotoxins isolated from $B$. jararacussu venom. Mice were pretreated with the extract $(500 \mathrm{mg} / \mathrm{kg})$ one hour before injection of BthTX-I or BthTX-II $(0.8 \mathrm{mg} / \mathrm{kg}$ of BthTX-I and $0.4 \mathrm{mg} / \mathrm{kg}$ of BthTX-II). Mice were killed after six hours and inflammatory exudates were withdrawn after washing the peritoneal cavity. Total leukocytes (A), PMN cells (B) and MN cells (C). Results are presented as mean $\pm \operatorname{SEM}(n=5) .{ }^{*} p<0.05$. 
Pereira IC et al. Anti-inflammatory activity of Blutaparon portulacoides ethanolic extract against the inflammatory reaction induced by Bothrops jararacussu venom and isolated myotoxins BthTX-I and II. J Venom Anim Toxins incl Trop Dis. 2009;15(3):538

\section{DISCUSSION}

Clinical investigations have demonstrated the efficacy of antivenoms in neutralizing life-threatening systemic effects associated with snakebites $(36,37)$. However, bothropic antivenom does not effectively neutralize the local effect induced by Bothrops venom $(38,39)$. Some alternatives have been proposed as coadjuvants of the antivenoms, including those based on the use of plant extract.

Vegetal extracts constitute an excellent alternative source of novel antiophidian agents. In many countries, vegetal extracts have been traditionally used in the treatment of envenomations caused by snakebites $(12-15,40)$. Therefore, several plants have already demonstrated antivenom activity $(2,7,11,41)$. However, in most cases, scientific proof of their antiophidian activity is still needed.

Envenomation by the $B$. jararacussu species leads to signs and symptoms similar to those provoked by other Bothrops species and emphasis has been placed on acute alterations such as hemorrhage, edema and necrosis (42). The present study showed that the venom induces paw edema that peaks one hour after venom injection. This result corroborates previous studies showing the ability of Bothrops venom to induce edema $(24,38,43)$.

B. portulacoides it is known popularly as "capotiraguá" and in folk medicine this plant has been employed to treat leucorrhea. In a previously phytochemical study the following compounds were isolated and identified within the hexane and ethanol extracts of $B$. portulacoides aerial parts: a methylenedioxyflavonol, the isoflavone irisone B and the steroids stigmasterol, sitosterol and campesterol (44). In an experimental model, the crude extract and some isolated compounds presented trypanocidal and leishmanicidal activity in vitro, and also antimicrobial activity (18).

In our experimental model, $B$. portulacoides ethanolic extract showed antiinflammatory effect against edema formation induced by crude venom and myotoxins, which were similar to dexamethasone. This result is similar to that found by Ticli et al. (24), using the same experimental model and venom, but with Cordia verbenacea methanolic extract. Also, Borges et al. (41) evidenced the ability of Casearia sylvestris extract (Flacourtiaceae) to inhibit edema-inducing activity of both whole venom and myotoxin II of Bothrops moojeni. Several reports have shown that plant-derived compounds, that act on arachidonic acid metabolism, lead to a marked inhibition of edema. Some of them have been characterized as inhibitors of 
Pereira IC et al. Anti-inflammatory activity of Blutaparon portulacoides ethanolic extract against the inflammatory reaction induced by Bothrops jararacussu venom and isolated myotoxins BthTX-I and II. J Venom Anim Toxins incl Trop Dis. 2009;15(3):539

cyclooxygenase and lipoxygenase activities (2). Additionally, some plants active against the lethal effect of snake venoms also act against the edema induced by venoms $(6,45)$.

In the present study, we also evaluated the capacity of $B$. portulacoides ethanolic extract to inhibit leukocyte migration to the peritoneal cavity induced by the venom or by studied myotoxins. Neutrophils play a vital role in host defense. They are usually the first cell type to reach the injury site and predominate numerically in a recent lesion. Neutrophil attraction and migration are effected by chemoattractants generated in the injured area (46). PMN and MN infiltration into the location of Bothrops inoculation was previously reported in the literature (47-51).

Our results demonstrated that $B$. jararacussu venom was capable of inducing a marked influx of leukocytes to the injection location with predominance of neutrophils. These data corroborate the results of Barbosa et al. (52) that showed the capacity of the $B$. jararacussu venom to recruit leukocytes to gastrocnemic muscle. We also evaluated the capacity of the myotoxins to induce an influx of leukocytes. The results had demonstrated that both BthTX-I, devoid of enzymatic activity and BthTX-II, enzymatically active, were able to induce leukocyte influx to the location of its injection. The literature reports the presence of infiltrated of PMN and MN after the injection of myotoxic PLA $\mathrm{A}_{2}$ from the venoms of Bothrops nummifer (53) and Bothrops jararacussu (54) in skeletal muscle of mice, and after intrapleural administration of myotoxins isolated from Bothrops jararacussu venom and Bothrops pirajai (32). Thus, it is possible to suggest that the $\mathrm{PLA}_{2}$ contained in $B$. jararacussu venom contributes to the leukocyte migration evoked by $B$. jararacussu venom.

The results also show that the $B$. portulacoides extract at the concentration of 500 $\mathrm{mg} / \mathrm{kg}$ did not reduce the leukocyte migration induced by the venom or by BthTX-II, whereas B. portulacoides extract at $500 \mathrm{mg} / \mathrm{kg}$ was capable of reducing the number of total leukocytes and polymorphonuclear cells induced by BthTX-I. It is possible that, in some sense, $\mathrm{PLA}_{2}$ activity is important for leukocyte migration, probably by inducing a more powerful release of inflammatory mediators necessary for leukocyte migration. In this aspect, the literature shows that PLA ${ }_{2}$ Asp49 is more powerful than the Lys49 in inducing inflammatory mediators such as IL-1, IL-6, TNF-a, beyond activating important adhesion molecules for the migration of leukocytes (55). 
From these investigations, it may be concluded that $B$. portulacoides ethanolic extract showed anti-inflammatory effect against $B$. jararacussu venom-induced edema formation. In addition, B. portulacoides ethanolic extract also inhibited inflammatory effects induced by isolated myotoxins from this venom, an action in which BThTX-I and II may participate. However, the migration induced by the venom and myotoxins was not inhibited by the extract, probably due to the fact that different mechanisms are involved in this event and in edema formation.

\section{REFERENCES}

1. Martz W. Plants with a reputation against snakebite. Toxicon. 1992;30(10): 113142.

2. Mors WB, Nascimento MC, Pereira BM, Pereira NA. Plant natural products active against snake bite - the molecular approach. Phytochemistry. 2000;55(6):627-42.

3. Phillipson JD, Anderson LA. Ethnopharmacology and Western medicine. J Ethnopharmacol. 1989;25(1):61-72.

4. da Silva JO, Coppede JS, Fernandes VC, Sant'ana CD, Ticli FK, Mazzi MV, Giglio JR, Pereira PS, Soares AM, Sampaio SV. Antihemorrhagic, antinucleolytic and other antiophidian properties of the aqueous extract from Pentaclethra macroloba. J Ethnopharmacol. 2005;100(1-2):145-52.

5. Izidoro LF, Rodrigues VM, Rodrigues RS, Ferro EV., Hamaguchi A, Giglio JR, Homsi-Brandeburgo MI. Neutralization of some hematological and hemostatic alterations induced by neuwiedase, a metalloproteinase isolated from Bothrops neuwiedi pauloensis snake venom, by the aqueous extract from Casearia mariquitensis (Flacourtiaceae). Biochemie. 2003;85(7):669-75.

6. Melo PA, do Nascimento MC, Mors WB, Suarez-Kurtz G. Inhibition of the myotoxic and hemorrhagic activities of crotalid venoms by Eclipta prostrate (Asteraceae) extracts and constituents. Toxicon. 1994;32(5):595-603.

7. Soares AM, Januário AH, Lourenço MV, Pereira AMS, Pereira PS. Neutralizing effects of Brazilian plants against snake venoms. Drugs Fut. 2004;29(11):1105-17.

8. De Fátima CBM, Cintra ACO, Veronese ELG, Lavrador MAS, Giglio JR, Pereira PS, Dias DA, Franca SC, Sampaio SV. Inhibition of the lethal and myotoxic activities of Crotalus durissus terrificus venom by Tabernaemontana catharinenses; identification of one of the active components. Planta Médica. 2000;66(5):424-8. 
9. Biondo R, Pereira AMS, Marcussi S, Pereira PS, França SC, Soares AM. Inhibition of enzymatic and pharmacological activities of some snake venoms and toxins by Mandevilla velutina (Apocynaceae) aqueous extract. Biochimie. 2003;85(10):101725.

10. Biondo R, Soares A, Bertoni B, França S, Pereira A. Direct organogenesis of Mandevilla illustris (Vell) Wodson and effects of its aqueous extract on the enzymatic and toxic activities of Crotalus durissus terrificus snake venom. Plant Cell Rep. 2004;22(8):549-52.

11. Borges $M H$, Soares AM, Rodrigues VM, Oliveira F, Fransheschi AM, Rucavado A, Giglio JR, Homsi-Brandeburgo MI. Neutralization of proteases from Bothrops snake venoms by the aqueous extract from Casearia sylvestris (Flacourtiaceae). Toxicon. 2001;39(12):1863-9.

12. Cavalcante WL, Campos TO, Dal Pai-Silva M, Pereira PS, Oliveira CZ, Soares AM, Gallacci M. Neutralization of snake venom phospholipase $A_{2}$ toxins by aqueous extract of Casearia sylvestris (Flacourtiaceae) in mouse neuromuscular preparation. J Ethnopharmacol. 2007;112(3):490-7.

13. Januário AH, Santos SL, Marcussi S, Mazzi MV, Pietro RCLR, Sato DN, Ellena J, Sampaio SV, Franca SC, Soares AM. Neo-clerodane diterpenoid, a new metalloprotease snake venom inhibitor from Baccharis trimera (Asteraceae): antiproteolytic and anti-hemorrhagic properties. Chem Biol Interact. 2004;150(3):243-51. 14. Oliveira CZ, Maiorano VA, Marcussi S, Sant'ana CD, Januário AH, Lourenço MV, Sampaio SV, Franca SC, Pereira PS, Soares AM. Anticoagulant and antifibrinogenolytic properties of the aqueous extract from Bauhinia forficata against snake venoms. J Ethnopharmacol. 2005;98(1-2):213-6.

15. Veronese EL, Esmeraldino LE, Trombone AP, Santana AE, Bechara GH, Ketulhut I, Cintra AC, Giglio JR, Sampaio SV. Inhibition of the myotoxic activity of Bothrops jararacussu venom and its two major myotoxins, BthTX-I and BthTX-II, by the aqueous extract of Tabernaemontana catharinensis A.DC. (Apocynaceae). Phytomedicine. 2005;12(1-2):123-30.

16. Siqueira JC. Amaranthaceae de Mata Atlântica. Acta Biol Leopold. 1990;1(12): 165-73.

17. Siqueira JC. Importância alimentícia e medicinal das Amaranthaceaes do Brasil. Acta Biol Leopol. 1987;9(1):5-22. 
18. Salvador MJ, Ferreira EO, Pral EMF, Alfieri SC, Albuquerque S, Ito IY, Dias DA. Bioactivity of crude extracts and some constituents of Blutaparon portulacoides (Amaranthaceae). Phytomedicine. 2002;9(6):566-71.

19. Gutiérrez JM. Understanding snake venoms: 50 years of research in Latin America. Rev Biol Trop. 2002;50(3):377-94.

20. Maiorano VA, Marcussi S, Daher MAF, Oliveira CZ, Couto LB, Gomes OA, Franca SC, Soares AM, Pereira PS. Antiophidian properties of the aqueous extract of Mikania glomerata. J Ethnopharmacol. 2005;102(3):364-70.

21. Pinho FM, Burdmann EA. Fatal cerebral hemorrhage and acute renal failure after young Bothrops jararacussu snake bite. Ren Fail. 2001;23(2):269-77.

22. Milani Junior R, Jorge MT, de Campos FP, Martins FP, Bousso A, Cardoso JL, Ribeiro LA, Fan HW, França FO, Sano-Martins IS, Cardoso D, Ide Fernandes C, Fernandes JC, Aldred VL, Sandoval MP, Puorto G, Theakston RD, Warrell DA. Snake bites by the jararacuçu (Bothrops jararacussu): clinicopathological studies of 29 proven cases in São Paulo State, Brazil. Q J Med. 1997;90(5):323-34.

23. Queiróz LS, Santo Neto H, Rodrigues-Simioni L, Prado-Franceschi J. Muscle necrosis and regeneration after envenomation by Bothrops jararacussu snake venom. Toxicon. 1984;22(3):339-46.

24. Ticli FK, Hage LI, Cambraia RS, Pereira PS, Magro AJ, Fontes MR, Stábeli RG, Franca SC, Giglio JR, Soares AM, Sampaio SV. Rosmarinic acid, a new snake venom phospholipase $A_{2}$ inhibitor from Cordia verbenacea (Boraginaceae): antiserum action potentiation and molecular interaction. Toxicon. 2005;46(3):318-27.

25. Gutiérrez JM, Lomonte $B$. Phospholipase $A_{2}$ myotoxins from Bothrops snake venoms. Toxicon. 1995;33(11):1405-24.

26. Andrião-Escarso SH, Soares AM, Rodrigues VM, Ângulo Y, Diaz C, Lomonte B, Gutiérrez JM, Giglio JR. Myotoxic phospholipases $A_{2}$ in Bothrops snake venoms: effect of chemical modifications on the enzymatic and pharmacological properties of bothropstoxin from Bothrops jararacussu. Biochimie. 2000;82(8):755-63.

27. Homsi-Brandeburgo MI, Queiroz LS, Santo-Neto H, Rodrigues-Simioni L, Giglio JR. Fractionation of Bothrops jararacussu snake venom: partial chemical characterization and biological activity of bothropstoxin. Toxicon. 1988;26(7):615-27. 28. Kini RM. Excitement ahead: structure, function and mechanism of snake venom phospholipase $A_{2}$ enzymes. Toxicon. 2003;42(8):827-40. 
29. Lomonte C, Martino R, Selvaggiolo M, Bona RM, Cazzato F, Milano R, Chiarulli G, Basile C. Calcitriol pulse therapy and histology of parathyroid glands in hemodialysis patients. J Nephrol. 2003;16(5):716-20.

30. Ownby CL, Selistre de Araújo HS, White SP, Fletcher JE. Lysine 49 phospholipase $A_{2}$ proteins. Toxicon. 1999;37(3):411-45.

31. Landucci EC, Castro RC, Pereira MF, Cintra AC, Giglio JR, Marangoni S, Oliveira B, Cirino G, Antunes E, De Nucci G. Mast cell degranulation induced by two phospholipase $\mathrm{A}_{2}$ homologues: dissociation between enzymatic and biological activities. Eur J Pharmacol. 1998;343(2-3):257-63.

32. De Castro RC, Landucci ECT, Toyama MH, Giglio JR, Marangoni S, De Nucci G, Antunes E. Leucocyte recruitment induced by type II phospholipase $A_{2}$ into the rat pleural cavity. Toxicon. 2000;38(12):1773-85.

33. Cecchini $A L$, Soares $A M$, Cecchini $R$, de Oliveira AH, Ward RJ, Giglio JR, Arantes EC. Effect of crotapotin on the biological activity of Asp49 and Lys49 phospholipases $A(2)$ from Bothrops snake venoms. Comp Biochem Physiol C Toxicol Pharmacol. 2004;138(4):429-36.

34. Cintra AC, Marangoni S, Oliveira B, Giglio JR. Bothropstoxin-I: amino acid sequence and function. J Protein Chem. 1993;12(1):57-64.

35. Van Arman CG, Begany AJ, Miller LM, Pless HH. Some details of the inflammations caused by yeast and carrageenin. $J$ Pharmac Exp Ther. 1965;150(2):328-34.

36. Cardoso JL, Fan HW, França FO, Jorge MT, Leite RP, Nishioka SA, Ávila A, Sano-Martins IS, Tomy SC, Santoro ML, et al. Randomized comparative trial of three antivenoms in the treatement of envenoming by lance-headed vipers (Bothrops jararaca) in São Paulo, Brazil. Q J Med. 1993;86(5):315-25.

37. Gutiérrez JM, Leon G, Rojas G, Lomonte B, Rucavado A, Chaves F. Neutralization of local tissue damage induced by Bothrops asper (terciopelo) snake venom. Toxicon. 1998;36(11):1529-38.

38. Picolo G, Chacur M, Gutiérrez JM, Teixeira CFP, Cury Y. Evaluation of antivenoms in the neutralization of hyperalgesia and edema induced by Bothrops jararaca e Bothrops asper snake venoms. Braz J Med Biol Res. 2002; 35(10):1221-8. 
39. Zamuner SR, Da Cruz-Hofling MA, Corrado AP, Hyslop S, Rodrigues-Simioni L. Comparison of the neurotoxic and myotoxic effects of Brazilian Bothrops venoms and their neutralization by commercial antivenom. Toxicon. 2004;44(3):259-71.

40. Mors WB. Plants active against snake bite. In: Wagner $\mathrm{H}$, Fansworth NR, editors. Economic and medicinal plant research; 1991. 353-73p. 5 vol.

41. Borges $\mathrm{MH}$, Soares $\mathrm{AM}$, Rodrigues VM, Andrião-Escarso $\mathrm{SH}$, Diniz $\mathrm{H}$, Hamaguchi A, Quintero A, Lizano S, Gutiérrez JM, Giglio JR, Homsi-Brandeburgo MI. Effects of aqueous extract of Casearia sylvestris (Flacourtiaceae) on actions of snake and bee venoms and on activity of phospholipases $A_{2}$. Comp Biochem Physiol B Biochem Mol Biol. 2000;127(1):21-30.

42. Gutiérrez JM, Leon G, Lomonte B. Pharmacokinetic-pharmacodynamic relationships of immunoglobulin therapy for envenomation. Clin Pharmacokinetics. 2003;42(8):721-41.

43. Olivo Rdo A, Teixeira CF, Wallace JL, Gutiérrez JM, Zamuner SR. Role of cyclooxygenases in oedema-forming activity of bothropic venoms. Toxicon. 2007;49(5):670-7.

44. Ferreira EO, Dias DA. A methylenedioxyflavonol from aerial parts of Blutaparon portulacoides. Phytochemistry. 2000;53(1):145-7.

45. Nuñez V, Otero R, Barona J, Saldarriaga M, Osorio RG, Fonnegra R, Jimenez SL, Dias A, Quintana JC. Neutralization of the edema-forming, defibrinating and coagulant effects of Bothrops asper venom by extracts of plants used by healers in Colombia. Braz J Med Biol Res. 2004;37(7):969-77.

46. Ferreira SH. Are macrophages the body's alarm cells? Agents Actions. 1980;10(3):229-30.

47. Gutiérrez JM, Chaves F, Cerdas L. Inflammatory infiltrate in skeletal muscle injected with Bothrops asper venom. Rev Biol Trop. 1986;34(2):209-19.

48. Safavi SM, Kazemi B, Esmaeili M, Fallah A, Modarresi A, Mir M. Effects of lowlevel He-Ne laser irradiation on the gene expression of IL-1 $\beta$, TNF- $\alpha$, IFN- $\gamma$, TGF- $\beta$, bFGF, and PDGF in rat's gingiva. Lasers Med Sci. 2008;23(3):331-5.

49. Teixeira CFP, Landucci ECT, Antunes E, Chacur M, Cury Y. Inflammatory effects of snake venom myotoxic phospholipases $A_{2}$. Toxicon. 2003;42(8):947-62. 
50. Teixeira CFP, Chaves F, Zamuner SR, Fernandes CM, Zuliani JP, Cruz-Hofling MA, Fernandes I, Gutiérrez JM. Effects of neutrophil depletion in the local pathological alterations and muscle regeneration in mice injected with Bothrops jararaca snake venom. Int J Exp Pathol. 2005;86(2):107-15.

51. Zamuner SR, Teixeira CFP. Cell adhesion molecules involved in the leukocyte recruitment induced by venom of the snake Bothrops jararaca. Mediators Inflamm. 2002;11(6):351-7.

52. Barbosa AM, Villaverde AB, Guimarães-Souza L, Ribeiro W, Cogo JC, Zamuner SR. Effect of low-level laser therapy in the inflammatory response induced by Bothrops jararacussu snake venom. Toxicon. 2008;51(7):1236-44.

53. Gutiérrez JM, Chaves F, Gene JA, Lomonte B, Camacho Z, Schosinsky K. Myonecrosis induced in mice by a basic myotoxin isolated from the venom of the snake Bothrops nummifer (jumping viper) from Costa Rica. Toxicon. 1989; 27(7):73545.

54. Gutiérrez JM, Nuñez J, Diaz C, Cintra AC, Homsi-Brandeburgo MI, Giglio JR. Skeletal muscle degeneration and regeneration after injection of bothropstoxin-II, a phospholipase $A_{2}$ isolated from the venom of the snake Bothrops jararacussu. Exp Mol Pathol. 1991;55(3):217-29.

55. Zuliani JP, Fernandes CM, Zamuner SR, Gutiérrez JM, Teixeira CFP. Inflammatory events induced by Lys-49 and Asp-49 phospholipases $A_{2}$ isolated from Bothrops asper snake venom: role of catalytic activity. Toxicon. 2005;45(3):335-46. 